\title{
Hydrothermal carbon enriched with oxygenated groups from biomass glucose as efficient carbocatalysts
}

\author{
Guodong Wen, ${ }^{[\mathrm{a}]}$ Bolun Wang, ${ }^{[\mathrm{a}]}$ Congxin Wang, ${ }^{[\mathrm{b}]}$ Zhijian Tian, ${ }^{[\mathrm{b}]}$ Robert Schlögl, ${ }^{[\mathrm{c}]}$ and Dang Sheng \\ $\mathrm{Su}^{\star[a, b]}$
}

\begin{abstract}
The synthesis of carbocatalysts enriched with specific functionalized groups is not only critical to study the mechanism, but is also important to prepare efficient carbocatalysts. In this study, metal-free carbocatalysts enriched with specific oxygenated groups with different morphology and size were synthesized from glucose via hydrothermal carbonization, in which cheap and widely available biomass could be converted to functionalized carbon using environmentally benign process. The hydroxyl and carbonyl enriched hydrothermal carbon (HTC) could be used in nitrobenzene reduction, and higher conversion could be obtained on the sphere morphology with smaller size. In Beckmann rearrangement of cyclohexanone oxime, carboxyl enriched HTC exhibited superior performance compared with some conventional solid acid (e.g., HY and HZSM-5), on which the strong acid sites and weak Lewis acid sites lead to high selectivity for the side product. Although the intrinsic acidity of carbon is weak, these results demonstrated that the carboxyl enriched carbon could be efficiently used in weak Brønsted acidcatalyzed reactions such as Beckmann rearrangement.
\end{abstract}

Carbon-based structures (e.g., carbon sphere and graphene) have aroused great attention in a wide variety of applications, such as catalyst support, adsorbents and electrode materials. ${ }^{[1]}$ Recently, it has been shown that carbon could be also used as important metal-free catalysts for many kinds of heterogeneous catalysts for sustainable chemistry, including thermocatalysis (e.g., dehydrogenation of ethylbenzene and light alkanes, ${ }^{[2]}$ oxidation of alcohol, ${ }^{[3]}$ oxidation of cyclohexane, ${ }^{[4]}$ epoxidation of styrene ${ }^{[5]}$ and reduction of nitrobenzene ${ }^{[6]}$ ), electrocatalysis,$^{[7]}$ and photocatalysis. ${ }^{[8]}$ However, the understanding of catalytic mechanism has been restricted because of the complicated carbon surface structures (e.g., defects, functional groups and metal impurities). ${ }^{[9]}$

The oxygenated groups have been assumed to be the active sites in many carbon-catalyzed reactions, ${ }^{[2,3 b, 6 a]}$ and the synthesis of carbon materials with specific oxygenated groups is regarded as an efficient and direct route to confirm the active site and study the mechanism. For example, we have confirmed that carbonyl groups are the active sites in the oxidative dehydrogenation reaction via synthesis of carbonyl groups enriched phenanthrenequinone cyclotrimer. ${ }^{[10]}$ This cyclotrimer exhibited obviously higher activity than other commercial carbon

[a] Dr. G.D. Wen, B.L. Wang, Prof. Dr. D.S. Su

Shenyang National Laboratory for Materials Science

Institute of Metal Research, Chinese Academy of Sciences

72 Wenhua Road, Shenyang 110016 (China)

E-mail: dssu@imr.ac.cn

[b] Dr. C.X. Wang, Prof. Z.J. Tian, Prof. Dr. D.S. Su

Dalian National Laboratory for Clean Energy

Dalian Institute of Chemical Physics, Chinese Academy of Sciences 457 Zhongshan Road, Dalian 116023 (China)

[c] Prof. Dr. R. Schlögl

Fritz Haber Institute of the Max Planck Society

Faradayweg 4-6, Berlin 14195 (Germany) materials and even metal phosphates and oxides. These results indicated that the synthesis of carbon enriched with specific functionalized groups is not only critical to study the mechanism but is also important to prepare highly efficient carbon catalysts. Titirici and coworkers have shown that cheap and widely available biomass could be converted to carbon materials via hydrothermal carbonization, which is simple, energy-effective and environmentally benign. ${ }^{[11]}$ Although the hydrothermal carbon (HTC) has been successfully applied in adsorption and electrocatalysis, the application in thermocatalysis is relatively less explored. Herein, the oxygenated groups enriched HTC with different size and morphology was synthesized and applied in the reduction of nitrobenzene and Beckmann rearrangement reactions. For hydroxyl and carbonyl groups enriched HTC, It was found that higher conversion for nitrobenzene reduction was exhibited on smaller sphere size. In Beckmann rearrangement of cyclohexanone oxime, carboxyl enriched HTC showed even better performance than some conventional solid acids (e.g., HY and HZSM-5) with strong acid sites.
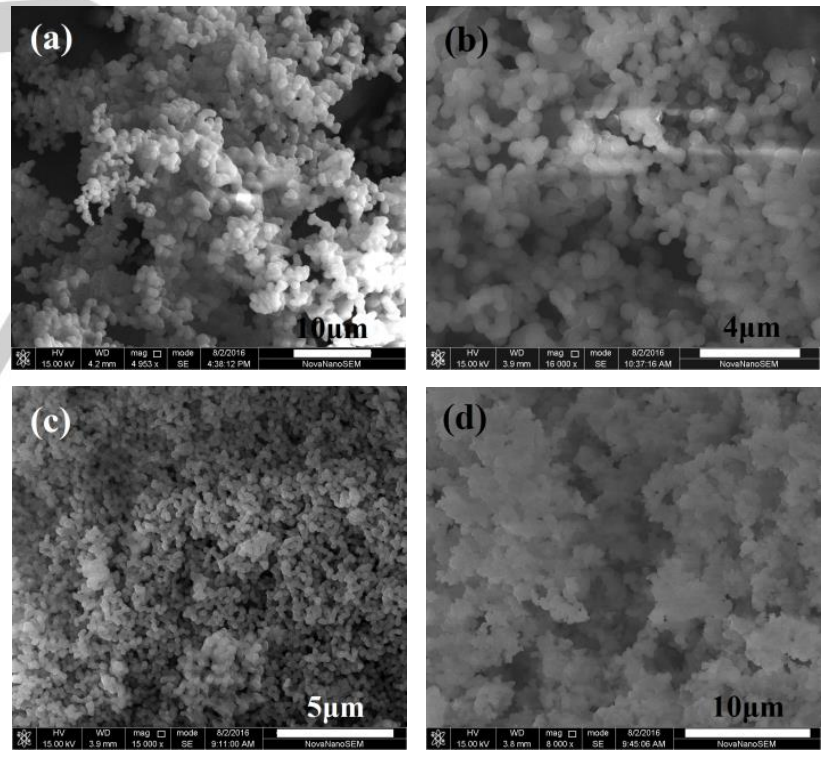

Figure 1. SEM images of HTC derived directly from glucose. (a) HTC-10G, (b) HTC-5G, (c) HTC-2.5G, (d) HTC-10GPVA.

The morphology of the HTC samples derived directly from glucose was shown in Figure 1 and Figure S1. It could be seen from these SEM (Figure 1) and TEM (Figure S1) images that carbon microspheres (ca. $560 \mathrm{~nm}$ ) were uniformly formed when the initial glucose concentration was $10 \mathrm{wt} \%$, and the average size of the spheres could be decreased to $227 \mathrm{~nm}$ as the initial glucose concentration was reduced to $2.5 \mathrm{wt} \%$. The addition of PVA could change the carbon sphere to small flake. All the HTC samples except the PVA dispersed HTC (denoted as HTC10GPVA) showed similar $I_{D 1} / I_{G}$ ratios (derived from Raman spectra, Figure S2), which were as high as ca. 1.8, indicating 
that the graphitization degree of the HTC was low. It is well known that biomass glucose is rich in oxygenated groups. As expected, the glucose derived HTC was also enriched with oxygenated groups, it was found from the XPS analysis that the oxygen atom concentration on the surface of HTC was as high as 13.0 at.\%. After deconvolution, we can see that the surface of $\mathrm{HTC}$ was enriched with $\mathrm{OH}$ and $\mathrm{C}=\mathrm{O}$ groups (Figure S3). Our previous work showed that the $\mathrm{OH}$ and $\mathrm{C}=\mathrm{O}$ groups could be used as active sites in the reduction of nitrobenzene reaction. ${ }^{[6 a]}$ Selective reduction of nitroarenes represents an important method to prepare corresponding aromatic amines with respect to both industrial application and academic research. ${ }^{[12]}$

Table 1. Reduction of nitrobenzene over HTC and other carbon catalysts. ${ }^{[a]}$

\begin{tabular}{lccc}
\hline Catalyst & Conv.\% & Aniline Sel. (\%) & $S_{\text {BET }}\left(\mathrm{m}^{2} / \mathrm{g}\right)$ \\
\hline Blank $^{[\mathrm{b}]}$ & 21.8 & 98.8 & - \\
HTC-10G & 47.1 & 100 & 6.5 \\
HTC-5G & 50.2 & 100 & 7.8 \\
HTC-2.5G & 61.5 & 94.1 & 17.0 \\
HTC-GPVA & 43.5 & 100 & 19.8 \\
UDD & 21.1 & 100 & 313.0 \\
Graphite & 37.7 & 96.1 & 10.8 \\
HHT & 19.5 & 100 & 33.8 \\
Activated carbon & 62.5 & 98.2 & 883.3 \\
\hline
\end{tabular}

[a] Reaction conditions: $20 \mathrm{mg}$ catalyst, $1.2 \mathrm{~g}$ nitrobenzene, 6.0 equivalent hydrazine monohydrate $(3.4 \mathrm{~g}), 100{ }^{\circ} \mathrm{C}, 4 \mathrm{~h}$. [b] Blank experiment was conducted in the absence of any catalyst.

When the initial glucose concentration for the preparation of HTC decreased from 10 to $2.5 \mathrm{wt} \%$, the nitrobenzene conversion using corresponding HTC as catalysts increased from 47.1 to $61.5 \%$ (Table 1), which was higher than that on other carbon catalysts such as UDD, HHT and graphite. Although activated carbon gave a bit higher conversion than HTC-2.5G, the surface area of activated carbon is nearly 52 times higher than that of HTC-2.5G. In other words, the activity of HTC-2.5G is obviously higher than that of activated carbon if the activity is normalized by surface area. Combined with SEM and TEM characterizations, it was assumed that smaller sphere size was beneficial for exposing more active sites, thus lead to superior conversion. This assumption was supported by the $\mathrm{N}_{2}$ physical adsorption analysis, which showed that the specific surface area increased from 6.5 to $17.0 \mathrm{~m}^{2} / \mathrm{g}$ when the initial glucose concentration decreased from 10 to $2.5 \mathrm{wt} \%$. It seemed that the small flake morphology due to the addition of PVA did not show positive influence on the reduction reaction. Four model molecules (cyclohexanol, cyclohexanone, 9-phenanthrol and anthrone) were used as model catalysts to study the effect of chemical environment on the activity of the two groups. It seemed that the carbonyl and hydroxyl groups attached to a $\mathrm{sp}^{3}$ carbon skeleton were not favorable for the reaction (Table S2).
Besides $\mathrm{OH}$ and $\mathrm{C}=\mathrm{O}$ groups, $\mathrm{COOH}$ group is also important among the various oxygenated groups. Carboxyl group can not only enhance the dispersion of carbon-supported metals, ${ }^{[13]}$ but can also be used as active center in some carbon-catalyzed reactions (e.g., acid-catalyzed reactions ${ }^{[14]}$ and oxidative coupling of amines to imines $\left.{ }^{[15]}\right)$. However, the concentration of carboxyl groups on HTC derived directly from glucose was low. Titirici and coworkers showed that carboxyl group could be enriched on HTC via adding acrylic acid in the carbonization process ${ }^{[16]}$ however the carboxyl content was not quantified and correlated with the adding amount of acrylic acid. Besides, they did not test the carboxyl enriched HTC as catalysts. Therefore, further study especially the quantification is necessary to optimize this process and resulting HTC. In this study, the content of carboxyl was quantified via Boehm titration (Table 2). The number of carboxyl groups increased obviously with the loading amount of acrylic acid raised. Even using minimum amount of acrylic acid (HTC-10G1A), the carboxyl concentration $(0.5 \mathrm{mmol} / \mathrm{g})$ was even higher than that on the CNTs refluxed in concentrated $\mathrm{HNO}_{3}\left(100 \mathrm{~mL} \mathrm{HNO}_{3}\right.$ per gram of CNTs) at $140{ }^{\circ} \mathrm{C}$, which was only $0.4 \mathrm{mmol} / \mathrm{g}$ in our previously reported work. ${ }^{[14]}$ Therefore, this process is efficient and environmentally benign compared to the $\mathrm{HNO}_{3}$ oxidation for the functionalization of carbon materials with carboxyl groups.

Table 2. Beckmann rearrangement of cyclohexanone oxime over carboxyl enriched HTC. ${ }^{[a]}$

\begin{tabular}{lcccc}
\hline Catalyst & $\begin{array}{c}\text { Conv. } \\
(\%)\end{array}$ & $\begin{array}{c}\text { Lactam } \\
\text { Sel. } \\
(\%)\end{array}$ & $\begin{array}{c}\text { Cyclohexanone } \\
\text { Sel. } \\
(\%)\end{array}$ & $\begin{array}{c}\text { Content of } \\
\text { carboxyl } \\
(\mathrm{mmol} / \mathrm{g})^{[\mathrm{b}]}\end{array}$ \\
\hline Blank $^{[\mathrm{c}]}$ & 10.3 & 72.8 & 27.2 & \\
HTC-10G & 13.9 & 94.5 & 5.5 & 0.3 \\
HTC-10G1A & 24.5 & 76.3 & 23.7 & 0.5 \\
HTC-10G3.3A & 29.1 & 74.5 & 25.5 & 0.6 \\
HTC-10G10A & 35.6 & 77.3 & 22.7 & 1.5 \\
HTC-10G10A300 & 49.1 & 89.1 & 10.9 & 2.0 \\
HTC-10G10A300m & 20.2 & 81.3 & 18.7 & 2.0 \\
HY & 53.3 & 26.2 & 58.9 & - \\
HZSM-5 & 22.8 & 0 & 52.0 & - \\
\hline
\end{tabular}

[a] Reaction conditions: $115 \mathrm{mg}$ cyclohexanone oxime, $10 \mathrm{~mL}$ benzonitrile, $50 \mathrm{mg}$ catalyst, $130{ }^{\circ} \mathrm{C}, 1.5 \mathrm{~h}$. [b] Determined by Boehm titration. [c] Blank experiment was conducted in the absence of any catalyst.

The surface of the carbon sphere is no longer smooth owing to the addition of acrylic acid (Figure 2 and Figure S4). The carboxyl enriched HTC was used as acid catalysts in Beckmann rearrangement of cyclohexanone oxime. This reaction is the key process for the production of caprolactam, which is an important intermediate for the manufacture of nylon- 6 . The industrial route for the reaction involves the application of concentrated $\mathrm{H}_{2} \mathrm{SO}_{4}$, which leads to pollution and corrosion. Therefore, a greener process is urgently needed. It was found that the conversion increased with the addition of acrylic acid from 0 to $10 \mathrm{wt} \%$ 
because of the increase of carboxyl content (Table 2). It was reported that the carboxyl content on HTC could be increased by the treatment at $300^{\circ} \mathrm{C}$ in air in a muff furnace..$^{[17]}$ Unfortunately, the conversion severely decreased from 35.6 to $20.2 \%$ (HTC$10 \mathrm{G} 10 \mathrm{~A} 300 \mathrm{~m}$ ), which was possibly due to the large microporous surface area (Table S1). The formation of microporosity was caused by the loss of strongly micropore-bound decomposition products during the heat treatment. ${ }^{[18]}$ We showed that the carboxyl groups could also be introduced by treatment in an open tube furnace (HTC-10G10A300). Fortunately, it seems that the formation of microporosity was suppressed when large amount of oxygen was introduced in this open tube furnace system. HTC-10G10A300 exhibited not only high conversion but also extremely high lactam selectivity as high as $89.1 \%$, which was even higher than some conventional solid acid catalysts such as HY and HZSM-5. Solid acid zeolite catalysts were widely studied in Beckmann rearrangement reactions. ${ }^{[19]}$ Although HY gave a bit higher conversion than HTC-10G10A300, much lower lactam selectivity was observed.
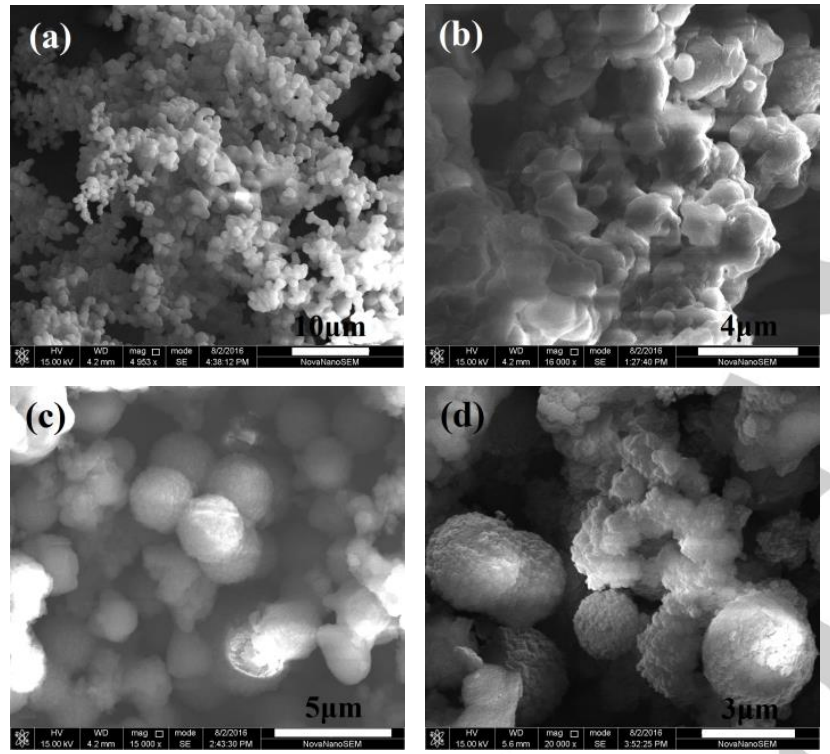

Figure 2. SEM images of carboxyl enriched HTC. (a) HTC-10G, (b) HTC10G1A, (c) HTC-10G3.3A, (d) HTC-10G10A, (e) HTC-10G10A300, (f) HTC10G10A300m.

$\mathrm{NH}_{3}$-TPD experiment was performed to compare the acidity between HTC sample and zeolite samples, the results were shown in Figure 3. A peak with extremely high intensity could be found on HTC-10G10A300 at low temperature (centered around $120^{\circ} \mathrm{C}$ ), indicating that large number of weak acid sites were presented on the HTC sample. However, strong acid sites on this HTC sample were generally negligible as almost no desorption signal was detected at higher temperature range. Although the peaks for HY and HZSM-5 at low temperature was much weaker than that on HTC, obvious peaks at higher temperatures were found, indicating the presence of strong acid sites on the two zeolites, which lead to the high selectivity for side product such as dimers or high-molecular weight byproducts. ${ }^{[19 c]}$ Moreover, extremely high selectivity for side product cyclohexanone was also observed on the two zeolites, which was likely caused by the known weak Lewis acid sites. ${ }^{[19 c]}$ These results demonstrated that carboxyl, which is a typical type of Brønsted acid site, could act as efficient and selective active center in the Beckmann rearrangement reaction.

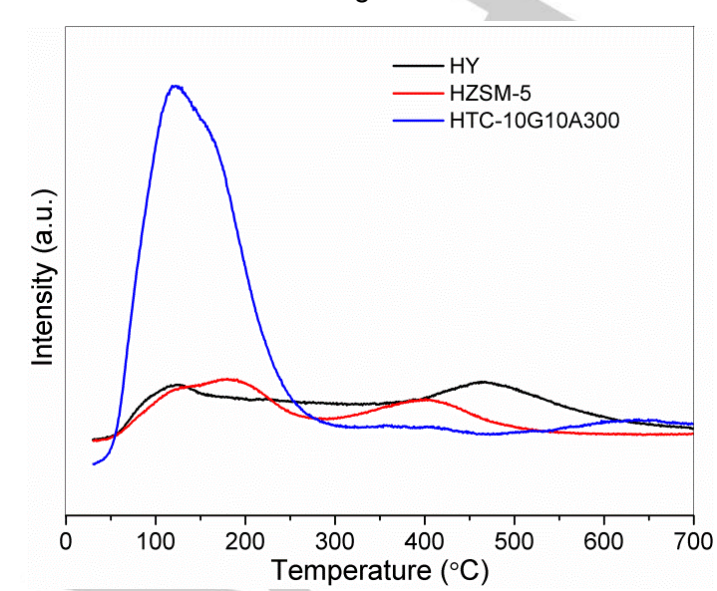

Figure 3. $\mathrm{NH}_{3}$-TPD profiles of HY, HZSM-5 and HTC-10G10A300.

Four model molecules (acetic acid, benzoic acid, 2naphthalenecarboxylic acid and 1-pyrenecarboxylic acid) were chosen to study the influence of chemical environment on the activity of carboxyl group. It seems that the carboxyl group attached to the $\mathrm{sp}^{3}$ carbon skeleton is not favorable for the formation of lactam (Table S3), and the large $\pi$-conjugation system is not favorable for the rearrangement either because the formation of lactam was suppressed with the increase of the benzene ring based on model molecules studies (Table S3). The graphitization degree of carboxyl enriched HTC is low, which could be demonstrated by the high $I_{D 1} / I_{G}$ ratios from the Raman spectra (Figure S5). It could be found from the TPD profiles that the desorption peak of carboxyl shifted to higher temperatures after treatment in air (Figure S6), indicating that the thermo stability of carboxyl groups increased after the treatment, thus the increase of the activity after treatment in air could be partially ascribed to the enhancement of the carboxyl stability.

In summary, oxygenated groups enriched HTC with different size and morphology could be successfully synthesized via hydrothermal carbonization. Hydroxyl and carbonyl groups enriched HTC could be used in nitrobenzene reduction reaction, and higher conversion could be obtained on carbon spheres with smaller size. Carboxyl enriched HTC could be applied in Beckmann rearrangement of cyclohexanone oxime. The number of surface carboxyls could be obviously increased by treatment in air in an open tube furnace. The performance of carboxyl enriched HTC was even better than conventionally used solid acids (e.g., HY and HZSM-5), which could be attributed to the significantly larger number of weak Brønsted acid sites on HTC, and the strong acid sites and weak Lewis acid sites on other solid acids are prone to lead to high selectivity of side product. Although the intrinsic acidity of carbon materials is weak, these results demonstrated that these weak Brønsted acid sites on carbon could exhibit significant advantages to other conventionally used solid acids in some weak Brønsted acidcatalyzed reactions such as Beckmann rearrangement. 


\section{Experimental Section}

All chemicals used were analytical grade. D-Glucose, anisole, hydrazine monohydrate (85\%), acrylic acid and activated carbon were supplied from Sinopharm Chemical Reagent Co., Ltd. Nitrobenzene and graphite (particle size 7-10 $\mu \mathrm{m}$ ) was purchased from Alfa Aesar. Ultradispersed nanodiamond (UDD) was bought from Beijing Grish Hitech Co. (China).. HHT (a typical CNT treated up to $2700^{\circ} \mathrm{C}$ ) was supplied from Pyrograf Products, Inc. $\mathrm{HZSM}-5\left(\mathrm{SiO}_{2} / \mathrm{Al}_{2} \mathrm{O}_{3}=60\right)$ and $\mathrm{HY}\left(\mathrm{SiO}_{2} / \mathrm{Al}_{2} \mathrm{O}_{3}=4.8\right)$ were provided from Dalian Institute of Chemical Physics, CAS.

To obtain hydrothermal carbon, $26 \mathrm{~mL}$ glucose aqueous solution was sealed into a Teflon inlet (volume $34 \mathrm{~mL}$ ) in an autoclave and treated in an oven at $190{ }^{\circ} \mathrm{C}$ for $16 \mathrm{~h}$. Then, the carbonaceous materials were washed with ethanol and dried at $80{ }^{\circ} \mathrm{C}$ overnight. The obtained hydrothermal carbon was denoted as $\mathrm{HTC}-\mathrm{xG}, \mathrm{x}$ represents the weight percentage of glucose in the initial solution. For the synthesis of carboxyl group enriched HTC, we added acrylic acid to the glucose aqueous solution. The glucose weight percentage was $10 \mathrm{wt} \%$, while the acrylic acid percentages were $1,3.3$, and $10 \mathrm{wt} \%$. The mixture was also transferred into the Teflon inlet, treated at $190{ }^{\circ} \mathrm{C}$ for $16 \mathrm{~h}$ and dried at 80 ${ }^{\circ} \mathrm{C}$ overnight. The obtained carboxyl group enriched HTC was marked as HTC-10GxA, $x$ is the weight percentage of the acrylic acid in the initial mixture. The HTC-10G10A sample was then calcined in air at $300{ }^{\circ} \mathrm{C}$ for $5 \mathrm{~h}$ in an open tube furnace or in a muff furnace, the obtained samples were denoted as HTC-10G10A300 and HTC-10G10A300m, respectively.

The surface area and pore volume were measured on Micrometrics ASAP 2020. Transmission electron microscopy (TEM) images were recorded on Tecnai $\mathrm{T} 12$ (FEI) with $120 \mathrm{kV}$ accelerating voltage. The particle size and morphology of the HTC was visualized from scanning electron microscopy (SEM) images by FEI Quanta FEG 250. The X-ray photoelectron spectroscopy (XPS) experiments were performed on ESCALAB 250 with a monochromatized Al Ka X-ray source. Raman experiments were conducted on LabRam HR 800 using 633 nm laser. The deconvolution of the spectra was similar to our previous work. ${ }^{[14]}$ Temperature-programmed desorption (TPD) was tested in $\mathrm{He}$ (50 $\mathrm{mL} / \mathrm{min}$ ) at a heating rate of $10{ }^{\circ} \mathrm{C} / \mathrm{min}$ from 80 to $900{ }^{\circ} \mathrm{C}$, the gas effluent was detected online with mass spectrometry (OmniStar GSD 300). $\mathrm{NH}_{3}$ TPD was carried out in $\mathrm{He}(50 \mathrm{~mL} / \mathrm{min})$ with $10^{\circ} \mathrm{C} / \mathrm{min}$ from 30 to $900^{\circ} \mathrm{C}$, the gas effluent was also analyzed online with mass spectrometry. Before the $\mathrm{NH}_{3}$ desorption, the samples were saturated adsorbed with $10 \%$ $\mathrm{NH}_{3} / \mathrm{Ar}(50 \mathrm{~mL} / \mathrm{min})$ for $40 \mathrm{~min}$ at room temperature. The carboxyl group was quantified by Boehm titration. Specifically, $0.1 \mathrm{~g}$ sample was immersed in $30 \mathrm{~mL} 0.01 \mathrm{~mol} / \mathrm{L} \mathrm{NaHCO}_{3}$ solution. After slowly stirred at room temperature for $24 \mathrm{~h}, 10 \mathrm{~mL}$ filtrate was pipetted, and the excessive base was titrated by $0.01 \mathrm{~mol} / \mathrm{L} \mathrm{HCl}$.

Nitrobenzene reduction was performed in a round-bottom flask in the presence of $1.2 \mathrm{~g}$ nitrobenzene and $3.4 \mathrm{~mL}$ hydrazine monohydrate over $20 \mathrm{mg}$ catalyst at $100{ }^{\circ} \mathrm{C}$ for $4 \mathrm{~h}$. The products were determined by GC (Agilent 7890) with HP-5 column, and anisole $(0.5 \mathrm{~g})$ was added as internal standard. Beckmann rearrangement of cyclohexanone oxime was conducted with $115 \mathrm{mg}$ cyclohexanone oxime and $10 \mathrm{~mL}$ benzonitrile over $50 \mathrm{mg}$ catalysts at $130^{\circ} \mathrm{C}$ for $1.5 \mathrm{~h}$. The products were analyzed by GC with HP-5 column, and DMF $(50 \mu \mathrm{L})$ was added as internal standard. The products were also identified with GC-MS (GC7890B-MSD5977, Agilent).

\section{Acknowledgements}

This work is financially supported by National Natural Science Foundation of China (No. 21503241, 21133010, 21261160487, 51221264, 21411130120, 21473223, 91545119, 91545110),
Strategic Priority Research Program of the Chinese Academy of Sciences (No. XDA09030103) and CAS/SAFEA International Partnership Program for Creative Research Teams.

Keywords: Carbocatalysts $\cdot$ hydrothermal $\cdot$ glucose $•$ nitrobenzene reduction $\cdot$ Beckmann rearrangement

[1] a) M. M. Titirici, R. J. White, N. Brun, V. L. Budarin, D. S. Su, F. de Monte, J. H. Clark, M. J. MacLachlan, Chem. Soc. Rev. 2015, 44, 250 290; b) J. Liu, N. P. Wickramaratne, S.Z. Qiao, M. Jaroniec, Nat. Mater 2015, 14, 763-774; c) P. F. Zhang, Z. A. Qiao, S. Dai, Chem. Commun. 2015, 51, 9246-9256.

[2] a) D. S. Su, S. Perathoner, G. Centi, Chem. Rev. 2013, 113, 57825816; b) J. Zhang, X. Liu, R. Blume, A. H. Zhang, R. Schlögl, D. S. Su, Science 2008, 322, 73-77.

[3] a) Y. M. Lin, D. S. Su, ACS Nano 2014, 8, 7823-7833; b) D. R. Dreyer H. P. Jia, C. W. Bielawski, Angew. Chem. 2010, 122, 6965-6968; Angew. Chem. Int. Ed. 2010, 49, 6813-6816; c) M. A. Patel, F. X. Luo, M. R. Khoshi, E. Rabie, Q. Zhang, C. R. Flach, R. Mendelsohn, E. Garfunkel, M. Szostak, H. X. He, ACS Nano 2016, 10, 2305-2315.

[4] H. Yu, F. Peng, J. Tan, X. W. Hu, H. J. Wang, J. Yang, W. X. Zheng, Angew. Chem. 2011, 123, 4064-4068; Angew. Chem. Int. Ed. 2011, 50, 3978-3982.

[5] a) Y. M. Lin, X. L. Pan, W. Qi, B. S. Zhang, D. S. Su, J. Mater. Chem. A 2014, 2, 12475-12483; b) A. Dhakshinamoorthy, A. Primo, P. Concepcion, M. Alvaro, H. Garcia, Chem. Eur. J. 2013, 19, 7547-7554

[6] a) S. C. Wu, G. D. Wen, X. M. Liu, B. W. Zhong, D. S. Su, ChemCatChem 2014, 6, 1558-1561; b) Y. J. Gao, D. Ma, C. L. Wang, J. Guan, X. H. Bao, Chem. Commun. 2011, 47, 2432-2434

[7] D. W. Wang, D. S. Su, Energy Environ. Sci. 2014, 7, 576-591.

[8] F. Z. Su, S. C. Mathew, G. Lipner, X. Z. Fu, M. Antoniett, S. Blechert, X C. Wang, J. Am. Chem. Soc. 2010, 132, 16299-16301.

[9] a) D. Chen, A. Holmen, Z. J. Sui, X. G. Zhou, Chin. J. Catal., 2014, 35, $824-841$; b) G. D. Wen, S. C. Wu, B. Li, C. L. Dai, D. S. Su, Angew. Chem. 2015, 127, 4178-4182; Angew. Chem. Int. Ed. 2015, 54, 41054109.

[10] J. Zhang, X. Wang, Q. Su, L. J. Zhi, A. Thomas, X. L. Feng, D. S. Su, R. Schlögl, K. Müllen, J. Am. Chem. Soc. 2009, 131, 11296-11297.

[11] a) M. M. Titirici, M. Antonietti, Chem. Soc. Rev. 2010, 39, 103-116; b) M. M. Titirici, R. J. White, C. Falco, M. Sevilla, Energy Environ. Sci. 2012, 5, 6796-6822

[12] a) R. V. Jagadeesh, A. E. Surkus, H. Junge, M. M. Pohl, J. Radnik, J. Rabeah, H. M. Huan, V. Schünemann, A. Brückner, M. Beller, Science 2013, 342, 1073-1076; b) Y. J. Gao, D. Ma, C. L. Wang, J. Guan, X. H. Bao, Chem. Commun. 2011, 47, 2432-2434.

[13] L. Y. Zhang, G. D. Wen, H. Y. Liu, N. Wang, D. S. Su, ChemCatChem 2014, 6, 2600-2606.

[14] G. D. Wen, J. Y. Diao, S. C. Wu, W. M. Yang, R. Schlögl, D. S. Su, ACS Catal. 2015, 5, 3600-3608.

[15] C. L. Su, M. Acik, K. Takai, K. Takai, J. Lu, S. J. Hao, Y. Zheng, P. P. Wu, Q. L. Bao, T. Enoki, Y. J. Chabal, K. P. Loh, Nature Commun. 2012, 3: 1298

[16] R. Demir-Cakan, N. Baccile, M. Antonietti, M. M. Titirici, Chem. Mater 2009, 21, 484-490

[17] Z. Chen, L. J. Ma, S. Q. Li, J. X. Geng, Q. Song, J. Liu, C. L. Wang, H. Wang, J. Li, Z. Qin, S. J. Li, Appl. Surf. Sci. 2011, 257, 8686-8691.

[18] S. A. Wohlgemuth, F. Vilela, M. M. Titirici, M. Antonietti, Green Chem. 2012, 14, 741-749.

[19] a) P. O'Sullivan, L. Forni, B. K. Hodnett, Ind. Eng. Chem. Res. 2001, 40, 1471-1475; b) C. Ngamcharussrivichai, P. Wu, T. Tatsumi, Appl. Catal. A 2005, 288, 158-168; c) C. Ngamcharussrivichai, P. Wu, T. Tatsumi, J. Catal. 2005, 235, 139-149. 


\section{COMMUNICATION}

Oxygenated groups enriched carbocatalysts with different size and morphology were synthesized via hydrothermal carbonization from glucose. The hydroxyl and carbonyl enriched hydrothermal carbon (HTC) could be used as catalysts in reduction of nitrobenzene, higher conversion could be obtained on smaller sphere size. The carboxyl enriched HTC could be efficiently used in weak Brønsted acid-catalyzed Beckmann rearrangement reaction.

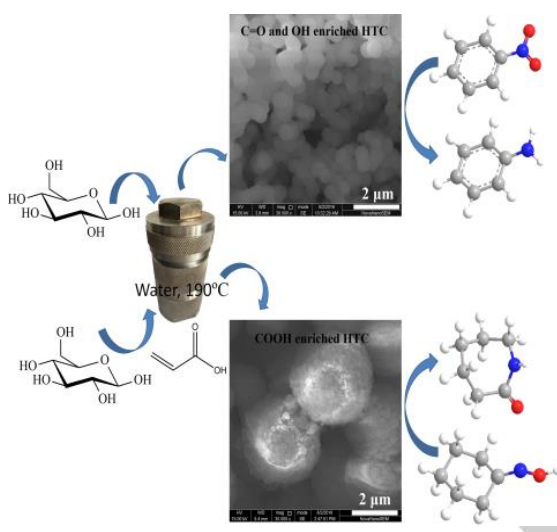

Guodong Wen, Bolun Wang, Congxin Wang, Zhijian Tian, Robert Schlögl, Dang Sheng Su*

Page No. - Page No.

Hydrothermal carbon enriched with oxygenated groups from biomass glucose as efficient carbocatalysts 